\title{
Information zu Gadolinium-Kontrastmitteln für die Magnetresonanztomografie
}

Eine Pressemitteilung des Berufsverbands Dt. Nuklearmediziner hat im Februar zu Irritationen und Verängstigungen der Patienten geführt. Die DRG hat eine umfassende wissenschaftliche Stellungnahme zu „gadoliniumhaltigen Kontrastmitteln in der Magnetresonanztomografie“ erarbeitet. Dieser Stellungnahme schließt sich der BDR vollumfänglich an.

Der BDR hat ergänzend eine Patienteninformation erarbeitet, die zur Auslage im Wartezimmer geeignet ist. Diese finden Sie nachfolgend dokumentiert, als Druckvorlage aber auch auf der BDR-Webseite, bzw. Sie können sie bei uns anfordern unter presse@radiologenverband.de

Ihr BDR

\section{Gadolinium-Kontrastmittel für die Magnetresonanztomografie \\ $\nabla$}

Sehr geehrte PatientInnen!

Die Magnetresonanztomografie (MRT) ist seit mehr als 2 Jahrzehnten eine sichere und unverzichtbare Bildgebungsmethode in der medizinischen Diagnostik. Sehr oft erübrigt sich durch die Auswahl der geeigneten Sequenzen eine zusätzliche Kontrastmittelgabe. Häufig ist aber zur weiteren Charakterisierung krankhafter Veränderungen die Gabe eines Kontrastmittels erforderlich. Dies verbessert die Aussagekraft in erheblichem und oft in entscheidendem Maße.

Derzeit sind in Deutschland verschiedene Kontrastmittelpräparate auf Basis von Gadolinium zugelassen. Diese Präparate werden überwiegend über die Nieren ausgeschieden. Insgesamt haben die verfügbaren MRT-Kontrastmittel eine sehr gute Verträglichkeit.
Allergische Reaktionen, als häufigste $\mathrm{Ne}$ benwirkung, werden nur selten beobachtet. Bei Patienten mit eingeschränkter Nierenfunktion gab es in der Vergangenheit bei wiederholten Kontrastmittelgaben in seltenen Fällen Anreicherungen in Geweben. Das hat zu erhöhter Achtsamkeit bei der Verabreichung dieser Präparate geführt. Deshalb erfolgt die Kontrastmittelgabe ausschließlich nach Überprüfung Ihrer persönlichen Risikofaktoren.

Zuletzt in der Presse verbreitete Meldungen über „Kontrastmittelablagerungen im Gehirn" haben vielfach zu Verunsicherung bei Patienten und kritischen Nachfragen geführt.

Schon seit etwa 2 Jahren ist bekannt, dass Gadolinium als Ablagerung in verschiedenen Regionen des Gehirns nachweisbar ist. Diese Anreicherungen wurden vor allem bei Patienten beobachtet, die bei Tumorleiden oder entzündlichen Erkrankungen wiederholt Kontrastmittel erhielten. Der derzeitige wissenschaftlichen $\mathrm{Er}$ -

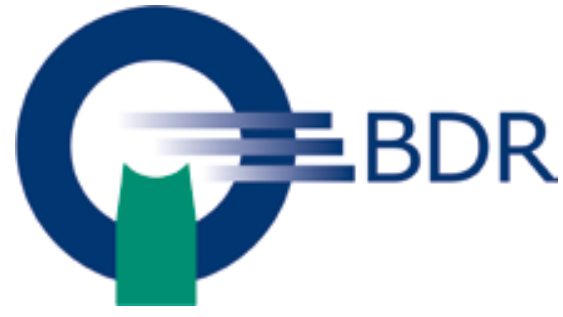

kenntnisstand zeigt zusammenfassend, dass diese Ablagerungen keine Erkrankungen oder Symptome verursachen.

Der untersuchende Radiologe/ die Radiologin wird unter Berücksichtigung Ihrer individuellen Krankengeschichte die sichere Auswahl des geeigneten Präparats treffen.

Zudem befolgen die Radiologen zu Ihrer Sicherheit folgende Grundsätze:

- Der Einsatz erfolgt nur nach sorgfältiger Abwägung, ob sich durch Kontrastmittel zusätzliche wichtige Informationen gewinnen lassen.

- Es wird grundsätzlich die geringste Menge eines Kontrastmittels verabreicht.

- Die Kontrastmittelgabe erfolgt nur unter Berücksichtigung ihrer individuell bestehenden Risikofaktoren (Nierenerkrankungen, Diabetes mellitus oder Allergien)

- Alle verwendeten Kontrastmittel sind nach internationalen und deutschen Richtlinien überprüft und als Arzneimittel zugelassen.

- Ihre Radiologin, ihr Radiologe stehen Ihnen selbstverständlich für weitere Fragen im persönlichen Aufklärungsgespräch gern zur Verfügung.

\section{Ihr Praxisteam}

